BOOK REVIEW

\title{
Learning Online: The Student Experience by George Veletsianos (2020). Baltimore, Maryland: Johns Hopkins University Press.
}

\author{
Reviewed by Tadd Kruse ${ }^{i}$
}

Technology continues to change the way in which we work, the way we socialise, and the way we learn under the expectation of a greater overall quality of life. Given the events of 2020 and especially the effects of the pandemic, our dependency on technology in higher education intensified. Many institutions pivoted from traditional learning settings to non-face-to-face instructional delivery methods largely facilitated through online learning mediums. In this book released in May 2020, George Veletsianos addresses the perceptions and reality of online learning settings across a myriad of perspectives and issues.

The book was written prior to the pandemic offering an in-depth look into the world of online education during a more normal period. Millions of students worldwide had engaged in online learning opportunities, and over the last 12 months those numbers have increased exponentially, making this timely and highly relevant for all tertiary educators. The author explores early on the value of online learning in comparison to face-to-face settings:

... the larger issue behind this debate is what conditions make for a high-quality educational experience, one that is effective, efficient, engaging, socially just, and meaningful and isn't exploitive, unfair or available only to those who can afford it ... ultimately, the value of an online course or degree rests upon its design and the strategies it employs rather than its modality. (p.18)

Online learning is ripe with critics questioning the authenticity, value, and credibility of this medium, and in this book Veletsianos argues that we must examine the learning experience through the lens of the student to fully appreciate this space.

Veletsianos addresses the intricacies of online learning from a student or learner experience vantage point, acknowledging the multiple roles or identities facing the modern student. The author is the Canada Research Chair in Innovative Learning and Technology and a professor in the School of Education and Technology at the Royal Roads University, a Canadian public university with its main campus in Colwood, British Columbia. George Veletsianos has published numerous journal articles and is also the author of the book Social Media in Academia: Networked Scholars.

i Dr Tadd Kruse is an Education Consultant, Membership Director for the International Association of Student Affairs and Services (IASAS) and a member of NASPA's (Student Affairs Administrators in Higher Education) Global Division and Middle East, North Africa and South Asia (MENASA) Advisory Boards. ORCid: 0000-0001-9916-9444. Email: taddkruse@gmail.com 
The text examines across 17 chapters the complicated realm of online learning, discusses the benefits and challenges within higher education settings, and suggests spaces to enhance effectiveness. Each in-depth chapter addresses online learning components ranging from effectiveness of the medium (student performance, attrition, growth of online learning, demographics, cheating, credibility), reasons students select online learning (flexibility, wider access, self-directed learning, massive open online courses or MOOCs), and interaction/performance factors (digital literacy, social support, social media, notetaking, motivation and socio-emotional factors). Throughout, Veletsianos highlights the intricacies of online learning and the need to overcome the disparities between learners, better known as the digital divide, all in a physically distant space.

The conciseness and organisation of the book, specifically the structure of the chapters, is one of the books greatest strengths. The chapter structures frame each topic via a concise and structured format first by introducing a story about single-student "learner" representing different characteristics or applied strategies in online learning, followed by a narrative digging deeper into the contributing factors. Each chapter is supported with relevant research and recognises limitations in existing research or data to propel the narrative. The referenced research in the field of online learning grounds the issues presented and provides resources for further research and consideration for educators. Finally, a summation of the perspectives are succinctly posed for consideration before closing with bulleted key points and a list of references. This concise and direct format allows educators to examine a single dimension of online learning within an empathetic real world student experience.

One area, and likely the only one, that lacks clarity throughout the book is the chapter titles. Each chapter is titled as "The Learner Who..." and presents as a listing of actions or student traits including nontraditional, self-directed, dropout, cheater, social networking user, and benefits from flexible learning. The chapter titles often allude to the topic, but are often vague so that, when flipping through the pages or looking over the table of contents, some targeted areas are not apparent without reviewing one's notes or scanning the bulleted items at the end of each chapter. More clear chapter titles would better serve educators as they consider this as a resource for the future, but may have detracted from the storybook approach to each chapter and the initial read - a small concession for a concise read that draws in the reader to the lived online learning student experience, the primary premise of the book.

Often when one hears of online learning, thoughts are immediately directed to a few characteristics of this educational capacity such as access, flexibility, or credibility issues. These immediate considerations fail to consider the multiple factors which (a) determine a student's reasons for selecting online learning options, and (b) influence the learning experience during one's educational journey. Learning Online: The Student Experience explores many of the issues confronting twenty-first century higher education and concisely presents major influences across the broad spectrum of online learning.

This book is an excellent resource for faculty and administrators to gain a broad understanding of the challenges and successes of online learning. Veletsianos, through his relaying 
of online learner experiences, presents the need for more intentional and integrated practices to make this medium more effective, mirroring steps often taken on campus to ensure greater student retention towards degree completion. Online learning presents in a multitude of forms, meeting a diverse range of needs for people around the world. The medium as a whole makes a significant contribution to society and higher education institutions. This book provides educators, regardless of geographic location, the scope to understand the complexities facing digital learning and at a timely point in history.

\section{How to cite:}

Kruse, T. (2021). Review on Learning Online: The Student Experience by George Veletsianos (2020). Baltimore, Maryland: Johns Hopkins University Press. Journal of Student Affairs in Africa, 9(1), 239-241. DOI: 10.24085/jsaa.v9i1.1443 